Commun. Fac. Sci. Univ. Ank. Series $\mathbf{A}_{1}$ V. 36 , pp 7-15 (1987)

\title{
ON FOUR DIMENSIONAL EINSTEIN MANIFOLDS
}

\section{HASAN ÖZEKES}

Faculty of Arts and Sciences

Istanbul Technical University, Istanbul, Turkey

(Received: March 1986, Accepted: 15 October 1986)

\section{ABSTRACT}

In this paper we provide a study of 4-dimensional Einstein manifolds, in particular we classify all 4-dimensional Einstein manifolds (up to local isometry) which have the property that tbe metric depends on only one coordinate.

Kasner (1923) considered the solutions of the Einstein equations involving functions of cnly one variable. His calculations are rather long and purely algebraic in nature. In this paper, by using a theorem of Singer and Thorpe (1969), we provije en alternative approach to find all 4-dimensional Einstein manitolds (up to local isometry) which have the property that the metric depends on only one coordinate. To do this we consider the metric

$$
\mathrm{d} \mathrm{s}^{2}=\mathrm{dt}^{2}+\sum_{\mathrm{i}=1}^{3} l_{\mathrm{i}}{ }^{2}(\mathrm{t}) \mathrm{dx}_{\mathrm{i}}{ }^{2}
$$

where $t$ is the arc-length and the following orthogonal recter fields:

$$
\mathbf{T}=\frac{\partial}{\partial \mathbf{t}}, \mathbf{X}_{1}, \mathbf{X}_{2} \text { and } \mathbf{X}_{3} \text { such that }|\mathbf{T}|=1,\left|\mathbf{X}_{\mathbf{i}}\right|=l_{1}(\mathbf{t}), \mathbf{i}=1,2,3
$$

Then, for the covariant differentiation $\nabla$, we ha, e

$$
\begin{aligned}
& \nabla_{\mathbf{x}_{\mathbf{i}}} \mathbf{X}_{\mathbf{j}} \cdot \mathbf{X}_{\mathrm{k}}=0, \nabla_{\mathbf{x}_{1}} \mathbf{X}_{\mathbf{j}} \cdot \mathbf{T}=0, \nabla_{\mathrm{T}} \mathbf{X}_{\mathbf{i}} \cdot \mathbf{X}_{\mathbf{j}}=0 \quad(\mathbf{i} \neq \mathbf{j}) \\
& \nabla_{\mathbf{x}_{\mathbf{i}}} \mathbf{X}_{\mathbf{i}} \cdot \mathbf{T}=-l_{\mathrm{i}} l^{\prime}{ }_{\mathrm{i}}, \nabla_{\mathbf{x}_{\mathbf{i}}} \mathbf{T} \cdot \mathbf{X}_{\mathbf{i}}=l_{\mathrm{i}} \underline{ }^{\prime}{ }^{\prime},
\end{aligned}
$$

so that $\nabla_{\mathrm{x}_{\mathbf{i}}} \mathrm{X}_{\mathrm{i}}=-l_{\mathrm{i}} l_{\mathrm{i}}^{\prime} \mathrm{T}, \nabla_{\mathrm{x}_{\mathrm{i}}} \mathrm{T}=\nabla_{\mathrm{T}} \mathrm{X}_{\mathrm{i}}=\frac{l^{\prime}{ }_{\mathrm{i}}}{l_{\mathrm{i}}} \mathrm{X}_{\mathbf{i}}, \nabla_{\mathbf{x}_{\mathbf{i}}} \mathrm{X}_{\mathrm{j}}=0(\mathrm{i} \neq \mathbf{j})$ and these determine the curvature as follows: 


$$
\begin{aligned}
& \mathbf{R}_{\mathbf{x}_{\mathbf{i}} \mathbf{x}_{\mathbf{j}}} \mathbf{X}_{\mathrm{k}}=0 \quad(\mathrm{i}, \mathrm{j}, \mathrm{k} \text { are distinct }),
\end{aligned}
$$

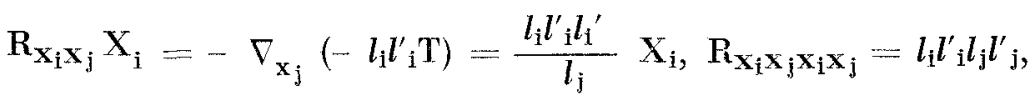

$$
\begin{aligned}
& \mathrm{R}_{\mathbf{x}_{\mathbf{i} \mathbf{x}_{\mathbf{j}}}} \mathrm{T}=\nabla_{\mathbf{x}_{\mathbf{i}}}\left(\frac{l_{\mathbf{j}}^{\prime}}{l_{\mathbf{j}}} \mathbf{X}_{\mathbf{j}}\right)-\nabla_{\mathbf{x}_{\mathbf{j}}}\left(\frac{l_{\mathbf{i}}^{\prime}}{l_{\mathbf{1}}} \mathbf{X}_{\mathbf{i}}\right)=0 \\
& \mathrm{R}_{\mathrm{x}_{\mathrm{i}} \mathrm{T}} \mathrm{X}_{\mathrm{i}}=\nabla_{\mathrm{x}_{\mathrm{i}}}\left(\frac{l_{\mathrm{i}}^{\prime}}{l_{\mathrm{i}}} \mathrm{X}_{\mathrm{i}}\right)+\nabla_{\mathrm{T}} l_{\mathrm{i}} l_{\mathrm{i}}^{\prime} \mathrm{T}=\left(-l_{\mathrm{i}}^{\prime 2}+l_{\mathrm{i}}{ }^{2}+l_{\mathrm{i}} l_{\mathrm{i}}{ }^{\prime \prime}\right) \mathrm{T}=l_{\mathrm{i}} l_{\mathrm{i}}{ }^{\prime \prime} \mathrm{T}, \\
& \mathrm{R}_{\mathbf{x}_{\mathrm{i}} \mathrm{Tx}_{\mathrm{i}} \mathrm{T}}=l_{\mathrm{i}} l_{\mathrm{i}}^{\prime \prime}, \mathrm{R}_{\mathbf{x}_{\mathrm{j}} \mathrm{T}} \mathrm{X}_{\mathrm{j}}=\nabla_{\mathrm{x}_{\mathrm{i}}}\left(\frac{l^{\prime}{ }_{\mathrm{j}}}{l_{\mathrm{j}}} \mathrm{X}_{\mathrm{j}}\right)=0(\mathrm{i} \neq \mathrm{j}) .
\end{aligned}
$$

Thus the sectional curvatures are

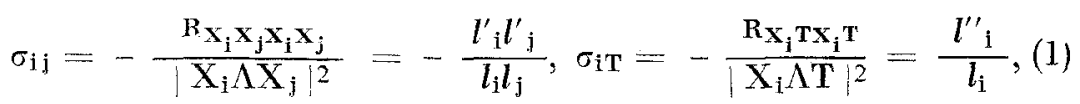

Now by the Theorem 1.4 of Singer and Thorpe (1969), the manifold $M$ with the above metric is Einstein if and only if

$$
\frac{l_{\mathrm{i}}^{\prime} l_{\mathrm{j}}}{l_{\mathrm{i}} l_{\mathrm{j}}}=\frac{l_{\mathrm{k}}^{\prime \prime}}{l_{\mathrm{k}}} \quad(\mathrm{i}, \mathrm{j}, \mathrm{k} \text { are distinct }) .
$$

Note that

$$
\left(\frac{l_{\mathrm{k}}^{\prime}}{l_{\mathrm{k}}}\right)^{\prime}=\frac{l_{\mathrm{k}}^{\prime \prime} l_{\mathrm{k}}-l_{\mathrm{k}}{ }^{2}}{l_{\mathrm{k}}^{2}}=\frac{l^{\prime \prime}{ }_{\mathrm{k}}}{l_{\mathrm{k}}}-\left(\frac{l_{\mathrm{k}}^{\prime}}{l_{\mathrm{k}}}\right)^{2}
$$

This and (2) show that if

$$
(\mathrm{u}, \mathrm{v}, \mathrm{w})=\left(\frac{l_{1}^{\prime}}{l_{1}}, \frac{l_{2}^{\prime}}{l_{2}}, \frac{l_{3}^{\prime}}{l_{3}}\right)
$$

then

$$
(u, v, w)^{\prime}=\left(v w-u^{2}, u w-v^{2}, u v-w^{2}\right) .
$$

The system of non-linear differential equations in (3), namely, the system.

$$
\begin{aligned}
& u^{\prime}=v w-u^{2} \\
& v^{\prime}=u w-v^{2} \\
& w^{\prime}=u v-w^{2}
\end{aligned}
$$


turns out to be much more easily solved than we might expect. This is because geometry tells us that each $(\mathrm{u}, \mathrm{v}, \mathrm{w})$ which satisfies (3) is tangent to the surface $u v+u w+v w=c(c=$ constant $)$, one reason for this is, since $-\mathrm{uv},-\mathrm{uw}$ and $-\mathrm{vw}$ denote the sectional curvatures, an Einstein manifold $M$ must have scalar curvature $-(u v+u w+r w)$ equal to some constant provided $\operatorname{dim} M \geqq 3$. Therefore the condition uv + uw $+v w=c$ reduces the system (4) from 3 -space to a surface. But further, since $v w=c-u v-u w$ we can write

similarly

$$
\begin{aligned}
& v w-u^{2}=c-u(u+v+w), \\
& u w-v^{2}=c-v(u+v+w), \\
& u v-w^{2}=c-w(u+v+w) .
\end{aligned}
$$

Thus

$$
(u, r, w)^{\prime}=(c, c, c)-(u+v-w)(u, v, w),
$$

which says that the tangent vector $(u, v, w)^{\prime}$ is in the plane through $(0,0,0),(1,1,1)$ and $(u, v, w)$, and the solution curve is the intersection of this plane $\mathbf{P}$ and the hyperboloid $u v+u w+v w=c$.

This argument shows that there is exactly one solution through each point of $I R^{4}$ and so we can find them. We now state the following

THEOREM: Let $M$ be a 4 -dimensional Einstein manifold whose metric depends on only one coordinate, that is, $M$ has the following metric

$$
\mathrm{ds}^{2}=\mathrm{dt}^{2}+l_{1}(\mathrm{t}) \mathrm{dx}^{2}{ }_{1}+l_{2}(\mathrm{t}) \mathrm{dx}_{2}{ }_{2}+l^{2}{ }_{3}(\mathrm{t}) \mathrm{dx}^{2}{ }_{3} .
$$

Then $\mathrm{M}$ is one of the following four types:

i) $l_{i}(t)=e^{a t+c_{i}}$ (a, $c_{i}$ constants) $i=1,2,3$ and thus $M$ is of constant sectional curvature (bence it is locally symmetric).

ii) $l_{i}(\mathrm{t})=c_{i}(\mathrm{t}+\mathrm{k})^{\mathbf{a}_{\mathrm{i}}+\mathrm{l} / 3}$ where $\mathrm{c}_{\mathrm{i}}>0, \mathrm{k}$ s.re constants and $a_{1}+a_{2}+a_{3}=0, a_{1}{ }^{2}+a^{2}{ }_{2}+a^{2}{ }_{3}=6$. The sectional curvatures are

$$
\sigma_{\mathrm{i} j}=-\frac{\left(\mathbf{a}_{\mathrm{i}}+1\right)\left(\mathbf{a}_{\mathbf{j}}+1\right)}{9(\mathbf{t}+\mathbf{k})^{2}} \mathrm{i}, \mathbf{j}=1,2,3, \mathbf{i} \neq \mathbf{j} .
$$

For $\left(a_{1}, a_{2}, a_{3}\right)=(2,-1,-1)$ (or its permutations) $M$ is flat. 
iii) $l_{i}(\mathbf{t})=c_{i}(\sin 3 t)^{1 / 3}(\csc 3 t-\cot 3 t)^{a_{i} / 3}$ where $c_{i}>0$ constant and $a_{i}$ 's are as in (ii). The sectional curvatures are

$$
\sigma_{i j}=-\frac{1}{s^{2}} \cdot\left[1-s^{2}+\left(\mathbf{a}_{\mathbf{i}}+\mathbf{a}_{\mathbf{j}}\right) \sqrt{1-\mathrm{s}^{2}}+\mathbf{a}_{\mathbf{i}} \mathbf{a}_{\mathbf{j}}\right]
$$

where $s=\sin 3 t$

iv) $l_{i}(t)=c_{i}(\sinh 3 t)^{1 / 3}(\tanh 3 t / 2)^{a_{i} / 3}$ where $c_{i}>0$ constant and $a_{i}$ 's are as in (ii). The sectional curratures are

$$
\sigma_{i j}=-\frac{1}{4 s^{2}\left(1+s^{2}\right)}\left(1+2 s^{2}+a_{j}\right)\left(1+2 s^{2}+a_{j}\right)
$$

where $s=\sinh (3 t / 2)$. For $\left(a_{1}, a_{2}, a_{3}\right)=(2,-1,-1), M$ is complete and analytic.

Procf: i) If the solution of (4) is constant, i.e. if the solution is on the diagonal of the cone (to which the hyperboloids are tangent), then we b.ave $u^{\prime}=v^{\prime}=w^{\prime}=0$ and from (4) it follows that $(u, v, w)=(a, a, a)$ ( $a=$ constant) is a solution. In this case $\iota_{i}=e^{a t+c_{i}}\left(c_{i}=\right.$ constant), and thus we obtain manifolds with constant sectional curvature $-a^{2}$. Since constant sectional curvature implies locally symmetric, these manifolds are locally symmetric.

ii) Note that by (4) and the fact that $u v+u w+v w=c$, we have

$$
\begin{aligned}
(u+v+w)^{2}=u^{2}+v^{2}+w^{2}+2(u v+u w+v w) & =c-\left(u^{\prime}+v^{\prime}+w^{\prime}\right)+2 c \\
& =3 c-\left(u^{\prime}+v^{\prime}+w^{\prime}\right) .
\end{aligned}
$$

Now if $c=0$, then we obtain $u+v+w=1 / t+k(k=$ constant $)$ and from $u^{\prime}=c-u(u+v+w)$ we get $u=a / t+k$ where a is constant. To determine $a$, we consider a vector $V=\left(a_{1}, a_{2}, a_{3}\right)$ such that

$$
\begin{aligned}
& a_{1}+a_{2}+a_{3}=0 \\
& a^{2}+a^{2}+a^{2}=6 .
\end{aligned}
$$

Then

$$
(\mathbf{u}, \mathrm{v}, \mathbf{w})=\left(\frac{\mathbf{a}_{1}+1}{3(\mathbf{t}+\mathrm{k})}, \frac{\mathrm{a}_{2}+1}{3(\mathrm{t}+\mathrm{k})}, \frac{\mathrm{a}_{3}+1}{3(\mathrm{t}+\mathrm{k})}\right)
$$

is a solution of (4) and it is on the cone. In this case $l_{i}(t)=$ $c_{i}(t+k)^{(a i+1) / 3}$ where $c_{i}>0$ constant, and the sectional curvatures are 


$$
\sigma_{i j}=-\frac{\left(a_{i}+1\right)\left(a_{j}+1\right)}{9(t+k)^{2}} \quad i, j=1,2,3 \text { and } i \neq j
$$

As $t$ approaches $-k$, the curvatures approach infinity, provided $a_{i} \neq-1 \neq a_{j}$. We note that the only integer soluticns of $(5)$ are $(-2,1,1)$ (and its permutations) and $(2,-1,-1)$ (and its permutations) in which case $\sigma_{\mathrm{ij}}$ become zero, so we obtain flat manifolds locally isometric to $\mathbb{R}^{4}$.

iii) If we let $V=\left(a_{1}, a_{2}, a_{3}\right)$ be as in (5), then $(u, v, w)=$ $\cot 3 \mathrm{t}(1,1,1)-\csc 3 \mathrm{t} \mathrm{V}$ is a solution curve which is outside the cone. In this case we have

$$
l_{\mathrm{i}}=\mathrm{c}_{\mathrm{i}}(\sin 3 \mathrm{t})^{1 / 3}(\csc 3 \mathrm{t}-\cot 3 \mathrm{t})^{\mathrm{a}_{\mathrm{i}} / 3} \text { where } \mathrm{c}_{\mathrm{i}}>0 \text { constant. }
$$

If we let $s=\sin 3 t$, then the sectional curvatures become

$$
\sigma_{i j}=-\frac{1}{s^{2}}\left[1-s^{2}+\left(a_{i}+a_{j}\right) \sqrt{1-s^{2}}+a_{i} a_{j}\right]
$$

and as $t$ tends to inf inity, s does not approach a number, so the curvatures do not approach a specific number.

iv) Let $V=\left(a_{1}, a_{2}, a_{3}\right)$ be as in (5). We note that $(u, v, w)=$ $\operatorname{coth} 3 \mathrm{t}(1,1,1)-\operatorname{csch} 3 \mathrm{tV}$ is also a solution curve which is inside the cone. In this case

$$
l_{\mathrm{i}}(\mathrm{t})=\mathrm{c}_{\mathrm{i}}(\sin 3 \mathrm{t})^{1 / 3}(\tanh 3 \mathrm{t} / 2)^{\mathrm{a}} \mathbf{i} / 3
$$

and the curvatures are

$$
\sigma_{i j}=-\frac{1}{4 s^{2}\left(1+s^{2}\right)}\left(1+2 s^{2}+a_{i}\right)\left(1+2 s^{2}+a_{j}\right)
$$

where $s=\sinh (3 t / 2)$. As $t$ tends to zero, $s$ tends tc zero and the curvatures approach infinity, provided $a_{i} \neq-1 \neq a_{j}$. If $a_{i}=-1=a_{j}$, that is, in the special case where $\left(a_{1}, a_{2}, a_{3}\right)=(2,-1,-1)$, as stends to zero $\sigma_{12} \rightarrow$ $-3 / 2$, and $\sigma_{23} \rightarrow 0$. Moreover as $\mathrm{s}$ tends to infinity all curvatures approach -1. Therefore this is the only case where for all critical values of $t$ the sectional curvatures are finite numbers. We will now show that the manifold $M$ with these sectional curvatures is complete and analytic.

For $t>0$ we consider the mapping of $M$ to $I R^{4}-\{0\}$ given by

$$
\left(t, x_{1}, x_{2}, x_{3}\right) \stackrel{\mu}{\rightarrow}\left(t \cos a x_{1}, t \sin a x_{1}, x_{2}, x_{3}\right) .
$$


We determine a so that we can extend the mapping to $\mathrm{IR}^{4}$ and make it a local isometry.

Observe that if $g$ is a smooth function, then

$$
g\left(y_{1}, y_{2}, y_{3}, y_{4}\right)=g\left(t \cos a x_{1}, t \sin a x_{1}, x_{2}, x_{3}\right)
$$

and so

$$
\begin{aligned}
& \frac{\partial \mathbf{g}}{\partial \mathrm{t}}=\cos \operatorname{ax}_{1} \frac{\partial \mathbf{g}}{\partial \mathbf{y}_{1}}+\sin \mathbf{a x}_{1} \frac{\partial \mathrm{g}}{\partial \mathbf{y}_{2}} \\
& \frac{\partial \mathbf{g}}{\partial \mathbf{x}_{1}}=- \text { at } \sin \mathbf{a x}_{1} \frac{\partial \mathbf{g}}{\partial \mathbf{y}_{1}}+\text { at } \cos \mathbf{a x}_{1} \frac{\partial \mathbf{g}}{\partial \mathbf{y}_{2}} \\
& \frac{\partial \mathbf{g}}{\partial \mathbf{x}_{2}}=\frac{\partial \mathbf{g}}{\partial \mathbf{y}_{3}} \\
& \frac{\partial \mathbf{g}}{\partial \mathbf{x}_{3}}=\frac{\partial \mathbf{g}}{\partial \mathbf{y}_{4}}
\end{aligned}
$$

From (6) we obtain the following vector fields

$$
\begin{gathered}
Y_{1}=\cos \operatorname{ax}_{1} T-\frac{\sin a x_{1}}{a t} X_{1} \\
Y_{2}=\sin \operatorname{ax}_{1} T+\frac{\cos a x_{1}}{a t} X_{1} \\
Y_{3}=X_{2}, \quad Y_{4}=X_{3} .
\end{gathered}
$$

Note that we have

$$
\begin{aligned}
& \mathrm{Y}_{1} \cdot \mathrm{Y}_{1}=\cos ^{2} \mathrm{ax}_{1}+\left(\frac{l_{1}}{\mathrm{at}}\right)^{2} \sin ^{2} \mathrm{ax}_{1} \\
& \mathrm{Y}_{1} \cdot \mathrm{Y}_{2}=\sin \mathrm{ax}_{1} \cdot \cos \mathrm{ax}_{1}-\left(\frac{l_{1}}{\mathrm{at}}\right)^{2} \sin \mathrm{ax}_{1} \cdot \cos \mathrm{ax}_{1} \\
& \mathrm{Y}_{2} \cdot \mathrm{Y}_{2}=\sin ^{2} \mathrm{ax}_{1}+\left(\frac{l_{1}}{\mathrm{at}}\right)^{2} \cos ^{2} \mathrm{ax}_{1} \\
& \quad \mathbf{Y}_{1} \cdot \mathrm{Y}_{3}=\mathrm{Y}_{1} \cdot \mathrm{Y}_{4}=\mathrm{Y}_{2} \cdot \mathrm{Y}_{3}=\mathrm{Y}_{2} \cdot \mathrm{Y}_{4}=\mathrm{Y}_{3} \cdot \mathrm{Y}_{4}=0 .
\end{aligned}
$$


If we choose a, so that

$$
\lim _{t \rightarrow 0} \frac{l_{1}}{a t}=1
$$

we then make $Y_{1}, Y_{2}, Y_{3}, Y_{4}$ orthogonal at $t=0$. From (8) it follows that $\mathbf{a}=3 \times 2^{-2 / 3} \mathbf{c}_{1}$.

Since

$$
\begin{gathered}
\sin \mathrm{ax}_{1}=\frac{\mathrm{y}_{2}}{\sqrt{\mathrm{y}_{1}^{2}+\mathrm{y}_{2}^{2}}}, \cos \mathrm{ax}_{1}=\frac{\mathrm{y}_{1}}{\sqrt{\mathrm{y}_{1}^{2}+\mathrm{y}_{2}{ }^{2}}}, \\
l_{1}=\mathrm{c}_{1} 2^{1 / 3} \mathrm{~s}\left(1+\mathrm{s}^{2}\right)^{1 / 6}
\end{gathered}
$$

and $s=\sinh (3 t / 2),(7)$ becomes

$$
\begin{aligned}
& Y_{1}, Y_{1}=\frac{y^{2} 1}{y^{2} 1+y^{2}{ }_{2}}+\frac{4}{9} \frac{y^{2}{ }_{2}}{\left(y^{2}{ }_{1}+y^{2}\right)^{2}} \sinh ^{2} \frac{3 \sqrt{y^{2}{ }_{1}+y^{2} 2}}{2} \\
& \cosh 1 / 3 \frac{3 \sqrt{y^{2} 1+y^{2} 2}}{2} \\
& Y_{1} \cdot Y_{2}=\frac{y_{1} y_{2}}{y^{2}{ }_{1}+y^{2}{ }_{2}}-\frac{4}{9} \frac{y_{1} y_{2}}{\left(y^{2}{ }_{1}+y^{2}{ }_{2}\right)^{2}} \sinh 2 \frac{3 \sqrt{y^{2} 1+y^{2} 2}}{2} \\
& \cosh ^{1 / 3} \frac{3 \sqrt{y^{2}{ }^{1}+y^{2} 2}}{2} \\
& \mathrm{Y}_{2} \cdot \mathrm{Y}_{2}=\frac{\mathrm{y}^{2}{ }_{2}}{\mathrm{y}^{2}{ }_{1}+\mathrm{y}^{2}{ }_{2}}+\frac{4}{9} \frac{\mathrm{y}^{2}{ }_{1}}{\left(\mathrm{y}^{2}{ }_{1}+\mathrm{y}^{2}{ }_{2}\right)^{2}} \sinh ^{2} \frac{3 \sqrt{\mathrm{y}^{2} 1+\mathrm{y}^{2} 2}}{2}
\end{aligned}
$$

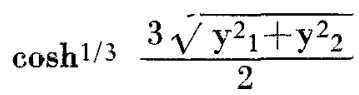

If we consider the power series expansions

$\sinh \mathbf{u}=\mathbf{u}+\frac{\mathbf{u}^{3}}{6}+\ldots, \quad \cosh \mathbf{u}=1+\frac{\mathbf{u}^{2}}{1}+\frac{\mathbf{u}^{4}}{24}+\ldots$

so that

$\sinh ^{2} u=u^{2}+\frac{u^{4}}{3}+\ldots, \cosh ^{1 / 3} u=1+\frac{u^{2}}{6}+\ldots$ 
the functions in (9) take the form

$$
\begin{aligned}
& \mathrm{Y}_{1} \cdot \mathrm{Y}_{1}=\frac{\mathrm{y}^{2} 1}{\mathrm{y}^{2}{ }_{1}+\mathrm{y}_{2}^{2}}+\frac{4}{9} \frac{\mathrm{y}^{2}}{\left(\mathrm{y}^{2}{ }_{1}+\mathrm{y}_{2}\right)^{2}}\left[\frac{9}{4}\left(\mathrm{y}^{2}+\mathrm{y}_{2}\right)+\right. \\
& \left.\frac{27}{16}\left(\mathrm{y}^{2} 1^{-} \mathrm{y}_{2}\right)^{2}+\ldots\right]\left[1+\frac{3}{8}\left(\mathrm{y}^{2}+\mathrm{y}^{2}{ }^{2}\right)+\ldots\right] \\
& Y_{1} \cdot Y_{2}=\frac{y_{1} y_{2}}{y^{2}+y^{2}}-\frac{4}{9} \frac{y_{1} y_{2}}{\left(y^{2}+y^{2}\right)^{2}}\left[\frac{9}{4}\left(y^{2}+y^{2}\right)+\right. \\
& \left.\frac{27}{16}\left(\mathrm{y}^{2}{ }_{1}+\mathrm{y}^{2}\right)^{2}+\ldots\right]\left[1+\frac{3}{8}\left(\mathrm{y}^{2}{ }_{1}+\mathrm{y}^{2} 2\right)+\ldots\right] \\
& \mathrm{Y}_{2} \cdot \mathrm{Y}_{2}=\frac{\mathrm{y}^{2} 2}{\mathrm{y}^{2} 1+\mathrm{y}^{2} 2}+\frac{4}{9} \frac{\mathrm{y}^{2} 1}{\left(\mathrm{y}^{2} 1+\mathrm{y}_{2}\right)^{2}}\left[\frac{9}{4}\left(\mathrm{y}^{2}+\mathrm{y}_{2}{ }^{2}\right)+\right. \\
& \left.\frac{27}{16}\left(\mathrm{y}^{2}{ }_{1}+\mathrm{y}^{2}{ }_{2}\right)^{2}+\ldots\right]\left[1+\frac{3}{8}\left(\mathrm{y}^{2}{ }_{1}+\mathrm{y}^{2}{ }_{2}\right)+\ldots\right]
\end{aligned}
$$

Note that these functions are defined and real analytic in IR ${ }^{4}$, therefore the manifold $M$, with the new metric, is real analytic.

We now shcw that $M$ is complete. For this we compare the lengths of the coordinate vector fields, that is, compare $1, l_{1}, l_{2}, l_{3}$ and 1 , at, 1,1 respectively. Under $\mu_{*}, X_{1}$ has the length at. This can be seen from the following 2-dimensional illustration.

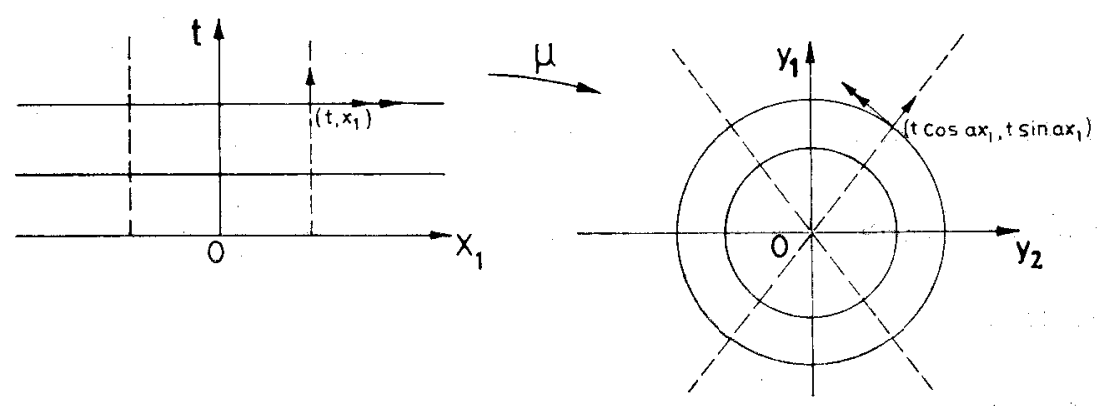

Fig. 1. 2 - dimensional illustratin of $\mu$ 
Note that at is approximately $(2 / 3)$ as $=c_{1} 2^{1 / 3} s \quad(s=\sinh 3 t / 2$, $\left.\mathrm{a}=3 \times 2^{-2 / 3} \mathrm{c}_{1}\right)$ and so $l_{1}=\mathrm{c}_{1}{ }^{2 / 3} \mathrm{~s}\left(1+\mathrm{s}^{2}\right)^{1 / 6} \geqq \mathrm{c}^{\prime}{ }_{1}$ at, where $\mathrm{c}^{\prime}{ }_{1} \geqq 1$ is some constant. Also $l_{2}=l_{3}=c_{2} 2^{1 / 3}\left(1+s^{2}\right)^{1 / 3} \geqq c_{2} 2^{1 / 3}$ provided $c_{2} \geqq 1$. Now let $c=\min \left(c_{2} 2^{1 / 3}, c^{\prime}{ }_{1}\right)$. Then $c \geqq 1$ and if $V=\Sigma a_{i} X_{i}$ is a vector,

$$
|\mathrm{V}|=\sqrt{\sum \mathbf{a}_{\mathrm{i}}^{2} \mathrm{~b}_{\mathrm{i}}^{2}} \geqq \sqrt{\overline{\sum \mathbf{a}_{\mathrm{i}}^{2} \mathbf{c}^{2}}}=|\mathbf{c}| \quad\|\mathbf{V}\|
$$

where $\|\cdot\|$ is the euclidean norm of $\mathrm{IR}^{4}$ and $|\cdot|$ is the norm on $\mathrm{M}$. Therefore the distance funsction on $M$ is bigger than or equal to the cuclidean distance in IR4. From this fact it follows that any Cauchy sequence in $M$ is a Cauchy sequence in $I R^{4}$. Since $I^{4}$ is complete, every Cauchy sequence converges. Therefore $M$ is complete.

\section{REFERENCES}

KASNER, E., (1923). Solutions of the Einstein Equations Involving Functions af only One Variable. Trans. Amer. Math. Soc. Vol. 27, 155-162.

KOBAYASHI, S. and NOMIZU, K., (1963) Foundatians of Differential Geometry, Vols. I, II. Interscience, New York,

SINGER, I.M. and THORPE, J.A., (1969) The Curvature of Four-Dimensional Einstein Spaces. Global Analysis. Papers in Honor of $K$. Kodaira, Princeton University Press, Princeton, 355-365.

TASHIRO, Y., (1981) Compact Four-Dimensional Einstein Manifolds, J. Differential Geometry, 16, 475-481.

WILLMORE, T. J., (1956) On Compact Riemannian Manifolds With Zero Ricci Curvature. Proc. Edinburg Math. Soc. (2), 10, 131-133. 